\title{
The Protein Kinase Receptor Modulates the Innate Immune Re- sponse against Tacaribe virus
}

\author{
Hector Moreno' ${ }^{1, *}$ and Stefan Kunz ${ }^{+}$ \\ 1 Institute of Microbiology, Lausanne University Hospital and University of Lausanne, Lausanne, Switzer- \\ land. \\ + Deceased on January 10 of 2020 \\ * Correspondence: Institute of Microbiology, Lausanne University Hospital, Lausanne CH-1011, Switzerland. \\ Phone: +41-21 31441 00, Fax: +41-21 314 4060, E-mail: hector.moreno@chuv.ch.
}

\begin{abstract}
The New World (NW) mammarenavirus group includes several zoonotic highly pathogenic viruses, such as Junin (JUNV) or Machupo (MACV). Contrary to Old World mammarenavirus, these viruses are not able to completely suppress the innate immune response, and trigger a robust interferon (IFN)-I response via retinoic acid-inducible gene I (RIG-I). Nevertheless, pathogenic NW mammarenaviruses trigger a weaker IFN response than their non-pathogenic relatives do. RIG-I activation leads to upregulation of a plethora of IFN-stimulated genes (ISGs), which exert a characteristic antiviral effect either as lone effectors, or resulting from the combination with other ISGs or cellular factors. The dsRNA sensor-protein kinase receptor (PKR) is an ISG that plays a pivotal role in the control of the mammarenavirus infection. In addition to its well-known protein synthesis inhibition, PKR further modulates the overall IFN-I response against different viruses, including mammarenaviruses. For this study, we employed Tacaribe virus (TCRV), the closest relative of the human pathogenic JUNV. Our findings indicate that PKR does not only increase IFN-I expression against TCRV infection, but also affects the kinetic expression and the extent of induction of Mx1 and ISG15 at both levels, mRNA and protein expression. Moreover, TCRV fails to prevent the effect of PKR on viral protein translation and its viral titer is inhibited when PKR is pre-stimulated via IFN-I. Here, we provide first evidence of the specific immunomodulatory role of PKR over selected ISGs, altering the dynamic of the innate immune response course against TCRV. IMPORTANCE: The mechanisms for innate immune evasion are key for emergence and adaptation of human pathogenic arenaviruses, and highly pathogenic mammarenaviruses such as JUNV or MACV trigger a weaker IFN response than non-pathogenic mammarenaviruses. Within the innate immune response context, PKR plays an important role in sensing and restricting the infection of TCRV virus. Although the mechanism of PKR for protein synthesis inhibition is well described, its immunomodulatory role is less understood. In this study, we found that TCRV protein expression and viral propagation are inhibited from early times after infection, and when externally activated, PKR inhibits TCRV viral progeny production. Our present findings further characterize the innate immune response in absence of PKR, unveiling the role of PKR in defining the ISG profile after viral infection.
\end{abstract}

Keywords: Protein Kinase Receptor (PKR); Mammarenavirus; Interferon; Innate immune response; Mx1; ISG15; CCL5.

\section{Introduction}

Mammarenaviruses are a large family of viruses divided in Old and New World arenavirus groups (OW and NW, respectively), according to antigenic properties, phylogeny, and geographic distribution (1). Both groups include zoonotic viruses that are highly pathogenic to humans, such as Lassa (LASV), JUNV, MACV, and Guaranito virus (2). The prototypic OW arenavirus Lymphocytic Choriominingitis virus (LCMV) is a neglected 
pathogen with a world-wide distribution and clinical significance in immunocompromised individuals and pregnant women (3). The highly diverse NW mammarenavirus group is further divided in four clades A, B, C and D, and while several members of Clade $B$ are confirmed human pathogens, some Clade D viruses show potential for viral emergence as well $(4,5)$. Mammarenaviruses are enveloped, bi-segmented, negative-stranded viruses with a life cycle restricted to the cytosol (6). The small genomic fragment encodes the glycoprotein precursor and the viral nucleoprotein (NP) while the large fragment codes for the matrix protein $(Z)$ and the RNA-dependent RNA-polymerase. The mammarenaviral NP participates in the replication/transcription complex $(7,8)$ and binds viral RNA genome, constituting the viral ribonucleoprotein.

Mammarenavirus infection is typically detected by RIG-I-like receptors and Toll-like receptors. The activation of these Pattern Recognition Receptors (PRRs) triggers an IFN-I immune response, upregulating a plethora of ISGs, which encode effector proteins that with antiviral effect and induce a cellular antiviral status. Mammarenaviruses are capable of inhibiting the IFN-I response to different extents, via a 5'-3' exoribonuclease NP domain (9-12), and highly pathogenic arenaviruses trigger a weaker induction of the IFN-I response than their non-pathogenic counterparts (12-16), suggesting that the capacity to overcome or suppress the IFN-I response is relevant for causing disease in humans. Indeed, the attenuated JUNV Candid\#1 triggers a stronger IFN- $\beta$ expression than the highly pathogenic Romero strain (17). Moreover, the $Z$ protein of pathogenic mammarenaviruses was shown to inhibit RIG-I, essential for the IFN-I response (16), summing to the immunosuppressive activity of NP. Despite the inhibitory effect of NP and Z over IFN-I response, and contrary to OW mammarenavirus, NW mammarenaviruses fail to completely abolish IFN-I response in human cells $(10,12,14,15,18)$.

In addition to the aforementioned host's PRRs, recent studies from others and us revealed that the dsRNA-PKR plays an important role during NW mammarenavirus infection $(14,18,19)$. PKR is an ISG that contributes to the enhancement of the IFN-I response against measles virus $(20,21)$, West-Nile virus (22), or upon IFN-I treatment (23). Upon detection and recognition of foreign dsRNA, PKR undergoes auto phosphorylation and subsequently phosphorylates the $\alpha$ subunit of eIF $2 \alpha$, leading to inhibition of protein capdependent translation (24). Interestingly, a recent study showed that, in contrast to LASV, highly pathogenic NW arenaviruses accumulate dsRNA during infections (25), possibly leading to the observed co-localization of the viral rNP with RIG-I, the melanoma differentiation-associated protein 5 (MDA5), and phosphorylated PKR $(19,26)$. Moreover, it has been shown that the highly pathogenic arenaviruses JUNV Romero Strain and MACV, but not LASV, induce higher IFN- $\beta$ levels in PKR null cells than in non-transduced parental cells (18). Nevertheless, previous results in our lab found show that PKR partially controls the infection by the non-human pathogenic TCRV but had no impact on the infection by the attenuated JUNV Candid\#1 strain (14).

Altogether, the literature suggests that the role of PKR in the IFN-I response might differ among pathogenic, attenuated, or non-pathogenic arenavirus infection. In the present study, we investigated and characterized the innate immune response triggered by non-pathogenic arenavirus TCRV in PKR KO cells, compared to parental cells subjected to an analogous mock CRISPR/Cas9 engineering. Our results indicate that PKR change the expression kinetic of Mx1 and ISG15, but not CCL5, whose expression is inhibited maintaining comparable pattern in presence and absence of PKR. Moreover, our findings indicate that activated PKR controls TCRV infection, inhibiting the viral progeny production and NP expression.

\section{Materials and Methods}

Cells, viruses and infections. Scrambled A549 control (A549/Scr) and PKR KO A549 (A549/PKR KO) cells were obtained as described in (14). Briefly, all cells were maintained in Dulbecco's modified Eagle medium with high glucose $(4.5 \mathrm{mg} / \mathrm{liter})$ and GlutaMAX (DMEM; Gibco BRL) with 10\% (vol/vol) fetal calf serum (FCS) and held in CO2 incubator 
$\left(37^{\circ} \mathrm{C}\right.$ and $5 \%$ (vol/vol) CO2). TCRV (strain 11573) was plaque purified and propagated in VeroE6 and Baby hamster Kidney (BHK) cells, followed by PEG-precipitation and sucrose cushion purification as described in (14).

For infections, cells were seeded $48 \mathrm{~h}$ in advance and counted before infection. The MOI was determined as described in each particular experiment. Before each experiment, cells were tested for mycoplasma contamination using a MycoAlert mycoplasma detection kit (Lonza. Basel, Switzerland). Inoculums were prepared by diluting the desired amount of virus in DMEM / 10\% FCS and incubated with cells for 90 minutes in CO2 incubator. Upon adsorption, inoculums were removed and fresh DMEM / 10\%FCS was added to each well. For infections in rIFN- $\alpha$ A/D-stimulated cells, 24 hours after seeding, cells were treated with $100 \mathrm{U} / \mathrm{ml}$ for 24 hours. Cells were then infected as described above.

Antibodies and reagents. Anti TCRV NP MA03-BE06 antibody (27) was obtained from BEI resources (Manassas, VA). Rabbit antibody against ISG15 was obtained from Cell Signaling Technology (Danvers, MA). Rabbit antibody against Mx1 was purchased from Proteintech (Rosemont, IL). Goat antibody against CCL5 was purchased from R\&D Systems (Minneapolis, MN).Antibody against Vinculin (EPR8185) was obtained from Abcam (Cambridge, UK). Recombinant human IFN (interferon- $\alpha$ A/D human; \#I4401) was purchased from Sigma-Aldrich (St. Louis, Missouri). Alexa Fluor-488 F(ab')2 fragment of goat anti-mouse IgG were obtained from Life Technologies (Carlsbad, CA). Polyclonal rabbit anti-mouse and donkey anti-goat antibody conjugated with horseradish peroxidase (HRP) were obtained from Dako (Santa Clara, CA).

Immunofocus assay (IFA). For viral titer quantitation by IFA, supernatants were cleared from cellular debris by centrifugation at $1200 \mathrm{rpm}$ for 3 minutes, and stored at $80^{\circ} \mathrm{C}$ until analysis. Samples were 10-fold serially diluted in DMEM / 10\% FCS and used to infect previously prepared VeroE6 cells in 96-well plate format. After 16-20h of infection, cells were washed with PBS, and fixed with $2 \%$ (wt/vol) formaldehyde for 30 minutes at room temperature. Then, cells were permeabilized with PBS / 0.1\% saponin / 1\% FCS (Working solution) for 30 minutes at room temperature. MA03-BE06 antibody was used as primary antibody to detect TCRV-NP (Sanchez et al., 1989), diluted 1:500 in working solution and applied to the cells for 1 hour at room temperature in a rocking station. Alexa 488-congugated anti-mouse IgG1 was used as secondary antibody, diluted 1:500 in working solution, and applied to cells for 45 minutes at room temperature in a rocking station. Before scoring the samples, cells were washed three times with PBS. Positive infectious foci were scored using an EVOS FLoid cell imaging station with a 20x Plan fluorite lens (Thermo Fisher).

RNA extraction, RT, qPCR and RT2 profiler. Samples collected for RNA extraction were kept in RNAlater (Sigma-Aldrich) at $-20^{\circ} \mathrm{C}$ until analysis. RNA for IFNb quantitation was extracted with a NucleoSpin RNA kit (Macherey-Nagel. Düren, Germany) and eluted in $60 \mu \mathrm{l}$ of nuclease-free water, in accordance with the manufacturer's instructions. RNA for RT2 profiler assay was extracted with TRIzol reagent (Invitrogen), following manufacturer's instructions and resuspending the RNA pellet in 20 microliters of nuclease-free water. Total RNA quantitation was performed with a Qubit 4.0 instrument (Thermo Fisher Scientific). 0.5 micrograms of total RNA were used for reverse transcription reactions using high-capacity cDNA reverse transcription kit (Applied Biosystems. Foster City, CA), following the manufacturer's instructions. TaqMan probes specific targeting human IFN- $\beta$ (Hs01077958_s1/FAM) and glyceraldehyde-3-phosphate dehydrogenase (GAPDH; Hs99999905_m1/VIC) were obtained from Applied Biosystems. Quantitative PCR (qPCR) was performed using a StepOne qPCR system (Applied Biosystems. Foster City, CA), and relative gene expression to GAPDH were determined following the $2-\Delta \Delta \mathrm{Ct}$ calculation (where $\mathrm{Ct}$ is threshold cycle). For transcriptome profiling, human antiviral response RT2 Profiler PCR array kits were used (PAHS-122ZG; Qiagen. Hilden, Germany). A total of $3.5 \mu \mathrm{g}$ (quantified by Qubit 4.0) of total cellular RNA were used for the reverse transcription reaction (RT2 SYBR green qPCR mastermix; Qiagen. Hilden, Germany). RT2 Profiler PCR array 384-well plates were set up by a PIRO personal pipetting robot 
(Labgene. Châtel-Saint-Denis, Switzerland). All RNA samples were tested for quality and integrity in a fragment analyzer (Agilent technologies. Santa Clara, CA), selecting only those with an RNA quality score (RIN) of 10. All tested samples were tested negative for genomic DNA contamination. Samples with aberrant amplification curves or shifted or multiple melting peaks were discarded from the analysis. qPCR reaction was performed using a LightCycler 480 instrument II (Roche. Basel, Switzerland) in accordance with the manufacturer's instructions, and relative gene expression to GAPDH were determined following the $2^{-\Delta \Delta \mathrm{Ct}}$ calculation (where $\mathrm{Ct}$ is threshold cycle).

Immunoblotting. Samples were collected and lysed in CelLytic by incubating on ice for 30 minutes. Then, samples were centrifuged for 15 minutes at $4^{\circ} \mathrm{C}$ at $14.000 \mathrm{rpm}$, supernatants moved to a new clean tube, and stored at $-80^{\circ} \mathrm{C}$ until analysis. Samples were then loaded with Laemli buffer in Novex Value $4 \%-20 \%$ Tris-Glycine pre-casted gels, and transferred to a nitrocellulose membrane. Membranes were stained with indicated antibodies diluted in $3 \%$ powder milk in PBS $0.1 \%$ Tween 20 . Signals were acquired by a ImageQuant LAS 4000 Mini (GE Healthcare Lifesciences), and quantitation and analyses of Western blot results were performed with ImageJ software.

Fluorescence-activated cell sorting (FACS). For TCRV-NP detection, cells were resuspended and fixed. Then cells were permeabilized with PBS / 1\% FCS (vol/vol) / 0.1\% Saponin $(\mathrm{wt} / \mathrm{vol})$ for $30 \mathrm{~min}$ at room temperature. After permeabilization, cells were washed once with PBS, stained with MA03-BE06 antibody (Sanchez et al., 1989) diluted 1:500, and incubated for 1 hour at room temperature. After primary antibody incubation, cells were washed once with PBS and incubated with Alexa-488-conjugated anti-mouse antibody (Life Technologies, Carlsbad, CA), diluted 1:500, for 45 minutes at room temperature. Cells were washed with PBS three times and scored using a FACSCalibur flow cytometer (Becton, Dickinson, San Jose, CA).

\section{Results}

To investigate the role of PKR in the IFN-I response triggered by NW mammarenavirus infection, we measured the levels of IFN- $\beta$ mRNA in control and PKR knockout human lung epithelial A549 cells (A549/PKR KO) infected with TCRV (strain 11573) and JUNV-Candid\#1. A549 cells have been extensively used by others and us to recapitulate mammarenavirus infection and represent a reliable model of study $(12,14,17,19)$. To obtain the A549/PKR KO cells, we used Clustered, regularly interspaced, short palindromic repeat-associated 9 (CRISPR/Cas9) guide RNAs (28, 29), and A549 cells subjected to analogous CRISPR/Cas9 editing with a scrambled guide RNA sequence (A549/Scr) as control cells. First, we infected A549/Scr and A549/PKR KO cells with TCRV at low multiplicity of infection (MOI. 0.01 PFU/cell) and collected total cellular RNA after 5 days of infection. We observed lower levels of IFN- $\beta$ transcripts in infected A549/PKR KO cells compared to infected A549/Scr control cells (Figure 1). These results are in agreement with previous studies on the role of PKR in the innate immune response against different viruses (20-22, 30 ), as well as with the previously reported reduced IFN-I response against JUNV compared to TCRV $(10,12,14)$. 


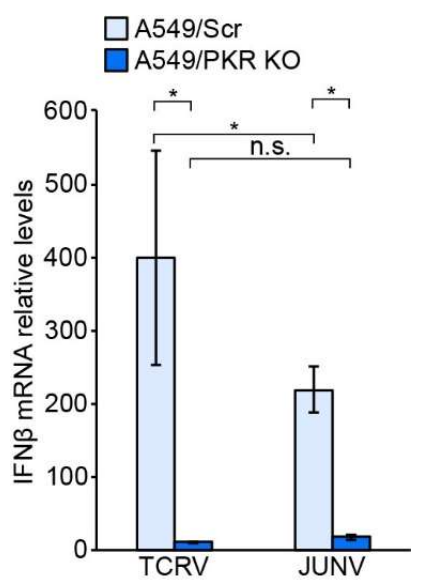

Figure 1. IFN $\beta$ mRNA expression in A549/Scr and A549/PKR KO cells infected with TCRV and JUNV. Cells were infected at MOI 0.01 PFU/Cell with TCRV and Junin Candid\#1. Total RNA was collected 5 days after infection and subjected to RT-qPCR as described in Material and methods. Fold-induction was calculated by the 2-๑®Ct method. Error bars represent standard deviations $(\mathrm{n}=6)$ and asterisks means statistical significance $(\mathrm{p}<0.01)$ in two-way ANOVA tests.

\begin{tabular}{|c|c|c|c|c|c|}
\hline CCL5 (6352) & $12673.81^{* *}$ & $5135.67^{\star \star *}$ & RELA (5970) & 2.1 & 0.84 \\
\hline IFNB1 (3456) & $5028.57^{\star *}$ & $2020.63^{\star *}$ & ATG5 (9474) & 1.87 & 1.61 \\
\hline MX1 (4599) & $1046.76^{\star \star}$ & $1753.85^{\star \star}$ & IRF3 (3661) & 1.86 & 1.72 \\
\hline ISG15 (9636) & $622.35^{\star *}$ & $751.67^{\star *}$ & АРОВЕССЗG (60489) & 1.82 & 1.45 \\
\hline OAS2 (4939) & $470.5^{\star \star}$ & $304.32^{\star *}$ & TBK1 (29110) & 1.76 & 2.85 \\
\hline IL6 (3569) & $142.55^{\star *}$ & $32.25^{\star \star}$ & RIPK1 (8737) & 1.74 & 0.81 \\
\hline IFIH1 (64135) & $56.58^{\star \star}$ & $62.8^{\star *}$ & CTSL (1514) & 1.72 & 2.27 \\
\hline IRF7 (3665) & $34.41^{\star \star}$ & $14.54^{*}$ & IL15 (3600) & 1.71 & 1.26 \\
\hline DDX58 (23586) & $30.11^{* *}$ & $38.48^{* *}$ & IL18 (3606) & 1.70 & 1.31 \\
\hline CXCL8 (3576) & $12.4^{\star \star}$ & $7.49^{\star \star}$ & CD80 (941) & 1.56 & 2.82 \\
\hline TLR3 (7098) & $10.65^{\star *}$ & $12.05^{\star *}$ & MAP2K1 (5604) & 1.54 & 1.99 \\
\hline IL12A (3592) & $9.34^{* *}$ & $5.27^{* *}$ & CASP10 (843) & 1.52 & 0.89 \\
\hline STAT1 (6772) & $9.25^{\star *}$ & $8.39^{* \star}$ & MAP3K7 (6885) & 1.49 & 1.48 \\
\hline CYLD (1540) & $5.67^{\star *}$ & $4.23^{* *}$ & $D D X 3 X(1654)$ & 1.41 & 1.06 \\
\hline TICAM1 (148022) & $3.7^{* *}$ & 1.23 & IRF5 (3663) & 1.41 & 1.10 \\
\hline NFKB1A (4792) & $3.49^{* *}$ & 2.69 & IFNAR1 (15975) & 1.37 & 1.05 \\
\hline IRAK1 (3654) & 3.23 & 0.77 & MAPK1 (5594) & 1.35 & 1.29 \\
\hline TRIM25 (7706) & $3.22^{\star *}$ & 1.64 & IKBKB (3551) & 1.30 & 0.64 \\
\hline NFKB1 (4790) & $3.17^{*}$ & 1.4 & MAPK8 (5599) & 1.30 & 1.00 \\
\hline MYD88 (17874) & $3.11^{* *}$ & $2.86^{* *}$ & CASP8 (841) & 1.26 & 1.08 \\
\hline TRADD (8717) & 2.94 & 2.54 & SPP1 (6696) & 1.21 & 207 \\
\hline CD40 (958) & 2.8 & 1.45 & PIN1 (5300) & 1.16 & 1.53 \\
\hline MAP2КЗ (26397) & $2.78^{\star *}$ & $1.96^{\star \star}$ & SUGT1 (10910) & 1.13 & 1.69 \\
\hline AZI2 (64343) & $2.73^{*}$ & 2.31 & HSP90AA1 (3320) & 1.05 & 1.27 \\
\hline TRAF6 (7189) & $2.7^{\star \star}$ & $1.78^{*}$ & CARD9 (64170) & 1.04 & 0.78 \\
\hline CHUK (1147) & $2.63^{* \star}$ & 2.37 & MAPK14 (1432) & 0.97 & 0.67 \\
\hline FADD (8772) & $2.63^{*}$ & 1.72 & MAPK3 (5595) & 0.93 & 0.45 \\
\hline CTSS (1520) & $2.41^{* *}$ & $2.61^{* *}$ & CTSB (1508) & 0.91 & 1.30 \\
\hline MAVS (57506) & $2.4^{\star \star}$ & 2.26 & IL1B (3553) & 0.85 & 0.83 \\
\hline TRAF3 (7187) & 2.36 & 1.34 & PYCARD (29108) & 0.73 & 1.13 \\
\hline CXCL9 (4283) & 2.13 & 3.21 & TKFC (26007) & 0.72 & 0.34 \\
\hline MAP3K1 (4214) & $2.12^{\star *}$ & 0.91 & FOS (14281) & 0.61 & 0.28 \\
\hline JUN (3725) & 2.11 & 0.59 & IFNA1 (3439) & 0.61 & 0.37 \\
\hline
\end{tabular}

Table 1. ISG profile of A549/Scr and A549/PKR KO cells infected with TCRV. Cells were infected at MOI of $0.01 \mathrm{PFU} / \mathrm{cell}$ and collected 5 days after infection. Values correspond to fold-induction (calculated by the 2๑๑Ct method) and asterisks means statistical significance $\left({ }^{*}: \mathrm{p}<0.01,{ }^{* *}: \mathrm{p}<0.001\right)$ in two-way ANOVA test. For clarity, color intensity is proportional to up- or down-regulation (red and blue, respectively). 
We recently showed that TCRV triggers a stronger innate immune response, yet comparable in the ISG profile, than JUNV-Candid\#1 (14). Thus, next we further characterized the IFN-I response in PKR null cells by studying the expression profile of ISGs. We compared the expression of 66 ISGs in A549/Scr and in A549/PKR KO cells infected with TCRV. For this aim, we performed infections at low MOI (0.01 PFU/cell) and collected cellular total RNA after 5 days of infection. Coherently with previous results (Figure 1), we observed that depletion of PKR in A549 cells reduces IFN- $\beta$ expression. The differences in the amplitude of IFN- $\beta$ expression between single IFN- $\beta$ RNA quantitation (Figure 1) and in the screening array (Table 1) are likely due to the use of different qPCR methods, as well as the employ of multiple housekeeping genes in the array, which renders a more accurate relative quantitation. Interestingly, despite lower IFN- $\beta$ mRNA levels, Mx1 and ISG15 increased their expression in A549/PKR KO cells (Table 1).

Given the relevance of these effectors in the IFN-I response, we monitored the protein levels of Mx1, ISG15, and CCL5 after TCRV infection. Our results show that the lack of PKR causes a delayed production of Mx1 and ISG15. However, concomitant with the higher gene expression observed (Table 1), Mx1 and ISG15 reach higher protein levels in A549/PKR KO cells than in A549/Scr control cells at late times after infection (Figure 2A). Moreover, absence of PKR resulted in overall inhibited CCL5 expression, without causing any delay in the expression kinetic after TCRV infection (Figure 2B). The effects of lacking PKR on the levels and dynamics expression of ISGs confirmed the key role of PKR in the innate immune response triggered by TCRV infection.

\section{A Vinculin
Mx1
$\begin{aligned} & \text { Ratio } \\ & \text { (vs. Vinc.) }\end{aligned}$
ISG15
$\begin{aligned} & \text { Ratio } \\ & \text { (vs. Vinc.) }\end{aligned}$ \\ B \\ Vinculin \\ CCL5 \\ Ratio} (vs. Vinc.)
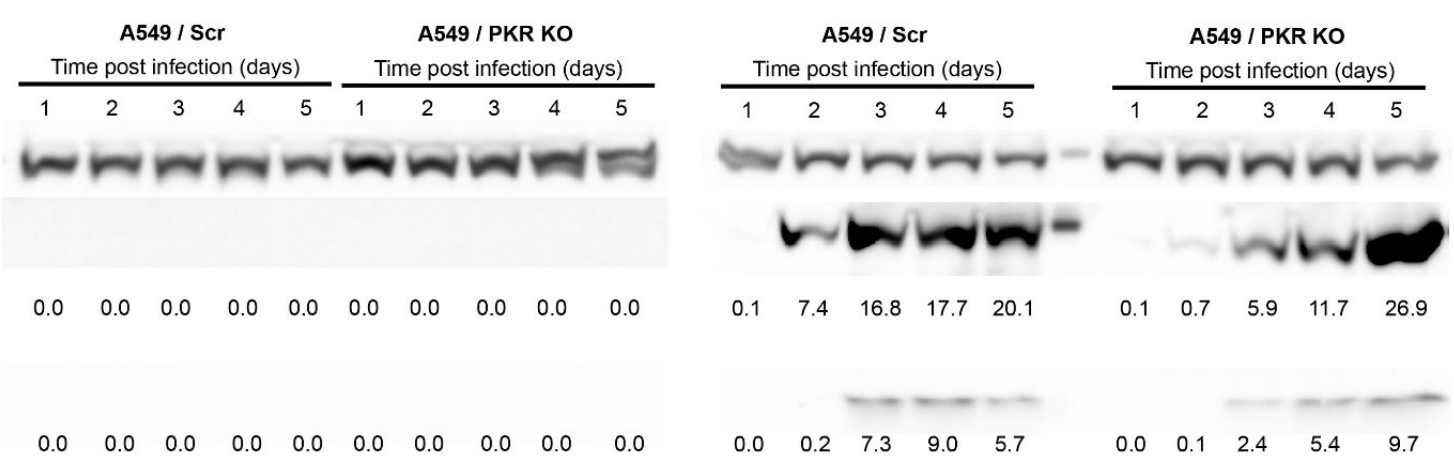

A549 / PKR KO Time post infection (days) $\quad$ Time post infection (days)

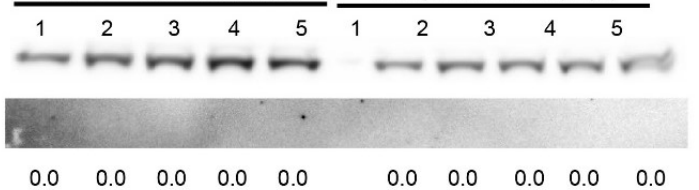
Figure 2. Protein expression of Mx1, ISG15 and CCL5 in TCRV infected A549/Scr and A549/PKR cells.
Cells were infected with TCRV at MOI of 0.01 and cell lysates were collected every 24 hours. Total cellular proteins were probed for Mx1, ISG15 (A) and CCL5 (B) expression by Western blotting. Vinculin was included as a loading control. The ratios of Mx1, ISG15 and CCL5 versus Vinculin were calculated by densitometry at the corresponding day post infection. One representative example out of three independent experiments is shown.

We next investigated the possible impact of PKR on viral protein expression over time. For this aim, we monitored TCRV NP expression by fluorescence-activated cell sorting (FACS), comparing A549/Scr and A549/PKR KO cells. We found higher expression levels of TCRV NP in A549/PKR KO cells than in control cells during the first 48h after infection, becoming comparable beyond that time point (Figure 3A). Coherently, we observed more efficient viral propagation in A549/PKR KO cells at early time points upon 
infection (Figure 3B). These results indicate that PKR limits viral propagation by reducing expression of viral proteins, at least during early stages of infection.
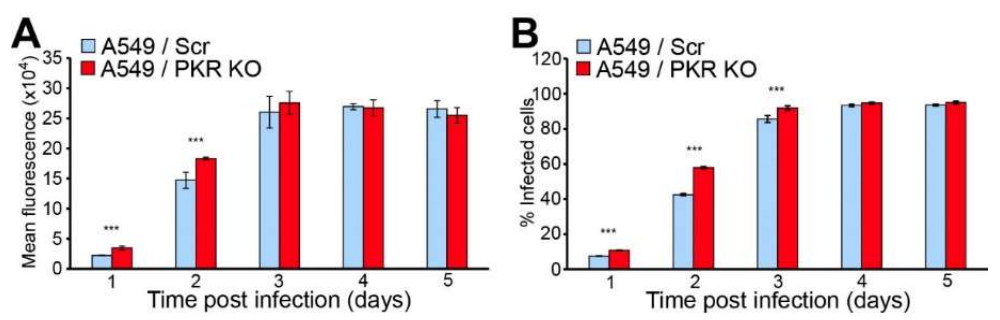

Figure 3. TCRV NP expression in A549/Scr and A549/PKR KO cells. Cells were infected with TCRV at MOI of 0.01 and samples were collected every 24 hours. Cells were stained for TCRV NP expression and corresponding florescent-tagged secondary antibody. Mean fluorescence of infected cells (A) and percentage of infected cells (B) are plotted. Percentage of infected cells was calculated using mock-infected cells as reference. Error bars represent standard deviations $(\mathrm{n}=3)$ and asterisks means statistical significance $(\mathrm{p}<0.01)$ in two-way ANOVA tests.

The results above strongly suggest that PKR activation efficiently controls TCRV infection. To further investigate that, we stimulated A549/Scr and A549/PKR KO cells with $100 \mathrm{IU} / \mathrm{ml}$ of $\mathrm{rIFN}-\alpha \mathrm{A} / \mathrm{D}$ and infected with TCRV at MOI of $0.01 \mathrm{PFU} / \mathrm{cell}$. Our results show that TCRV reach significantly higher viral titers in the absence of activated PKR than in its presence during early times after infection ( $<3$ days post infection), albeit production of viral progeny becomes comparable at later time points (Figure 4 ). These results indicate that, once activated, PKR can partially restrict TCRV growth.

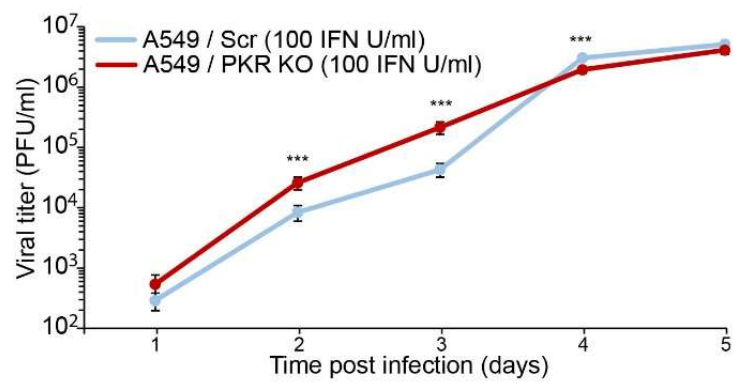

Figure 4. TCRV viral progeny production in IFN-stimulated A549/Scr and A549/PKR KO cells. Cells were stimulated with $100 \mathrm{IU} / \mathrm{ml}$ of rIFN- $\alpha \mathrm{A} / \mathrm{D} 24$ hours before infection. Cells were infected at MOI $0.01 \mathrm{PFU} / \mathrm{cell}$ and supernatants were assayed for viral titer by IFA. Error bars represent standard deviations $(n=4)$ and asterisks means statistical significance $(\mathrm{p}<0.01)$ in two-way ANOVA test.

\section{Discussion}

In the present study, we show that, despite causing a reduced expression of IFN- $\beta$ (Figure 1), depletion of the dsRNA sensor PKR leads to changes in the levels and dynamics of production of ISGs, including Mx1 and ISG15 (Table 1, Figure 2). Previous reports already described PKR as an enhancer of the IFN-I response upon viral infection $(20-22,30)$. Interestingly, in the case of JUNV Candid\#1, despite the differences in IFN- $\beta$ mRNA levels in infected A549/Scr and A549/PKR KO cells, viral progeny production was not affected (14), suggesting that either the contribution of PKR against JUNV infection is not biologically relevant or that JUNV can deploy molecular mechanisms to overcome host's innate immune response. Interestingly, a previous study showed that infections with the highly pathogenic JUNV Romero strain and MACV at high MOI (3 PFU/cell) in non-transduced A549 cells and A549/PKR KO cells results in an increase of the IFN- $\beta$ expression (18); suggesting that attenuated and pathogenic JUNV strains may interact with PKR in different manners or efficiencies. 
We previously described that TCRV infection is increased in A549/PKR KO cells at late time points, concomitantly with PKR activation in A549 cells (14). Here we also found that PKR partially controls the expression of TCRV NP during early stages of the infection (Figure 3), suggesting that PKR may also be activated during initial virus propagation. In this scenario, PKR could inhibit the early local viral propagation and viral protein synthesis, remaining undetectable by analysis of whole cell lysate, as only a small fraction of PKR is activated. Indeed, during infections performed in IFN-stimulated cells, TCRV reached higher viral titers in A549/PKR KO cells at early times than in control cells (Figure 4). This observation suggests that, despite being able to affect initial viral protein production, the impact on viral progeny production can only occur with extensive PKR activation, either via induced IFN stimulation or via intrinsic viral detection.

Despite rendering lower IFN- $\beta$ levels, TCRV infection in A549/PKR KO cells results in a delay of Mx1 and ISG15 expression, but also in increased mRNA and protein expression of both host factors at late time points (Table 1 and Figure 2). Noteworthy, reduced ISG15 and Mx1 expression occurs concomitantly with increased TCRV NP expression (Figures 2 and 3), suggesting that the observed antiviral effect of PKR may be implemented with the participation of these ISGs. In contrast, CCL5 expression is reduced because of the absence of PKR at both, gene and protein levels, and renders a comparable kinetic of expression. Therefore, PKR ablation seems to alter the ISG profile kinetic of Mx1 and ISG15, but not other ISGs, such as CCL5. Our results then suggest that the observed differences in viral progeny production in TCRV but not in JUNV (14) might be the result of not only reduced IFN- $\beta$ levels but also of changes in the expression pattern of specific ISGs and altered innate immune response.

CCL5 is a chemokine expressed in many cell types in response to viral infections and IFN- $\beta$, and plays a pivotal role in migration of effector and memory T cells $(31,32)$. CCL5 is a relevant player in the response against arenaviruses, and its absence in mice leads to establishment of chronic infections of LCMV clone 13 (33). Moreover, infection with the non-human pathogenic NW mammarenavirus Pichinde virus (PICV) p2 strain, which causes mild disease in guinea pigs, was followed by increased CCL5 expression at late time points, in contrast to the virulent PICV p18 strain (34). The CCL5 reduction due to the lack of PKR may then contribute to worse disease outcome in mammarenavirus infections. In such scenario, the weaker PKR activation observed in highly pathogenic NW mammarenaviruses lead contribute to a lower CCL5 expression and increased virulence.

ISG15 is strongly induced by the IFN-I cascade and exert its antiviral activity by being incorporated to nascent peptides in a process, similar to ubiquitination, termed ISGylation, which affects their stability (35). The expression of ISG15 is increased in response to many viral infections, including influenza A, Ebola, hepatitis B and C, human immunodeficiency virus 1, human papillomavirus, West Nile, and Zika (36). Nevertheless, many other viruses, such as Middle East and severe acute respiratory syndromes, foot and mouth disease virus, or Influenza B, deploy mechanisms to prevent the antiviral effect of ISG15 (37-41). Interestingly, besides its role as effector ISG, ISG15 also prevents the overamplification of the IFN-I cascade (40). It is then plausible that the lower expression of ISG15 in the absence of PKR at early time points upon TCRV infection disrupts the regulatory feedback loop, failing to repress the IFN-I response at later times, which may result in increased expression of Mx1 and ISG15.

Mx1 is an effector ISG with GTPase activity and antiviral effect against several viral infections, including Influenza, bunyaviruses, and hantaviruses (42-45). When activated, Mx1 oligomerizes and sequesters viral factors of LaCrosse virus, Influenza A or Thogoto virus, disturbing the viral life cycle $(42,45,46)$. Unlike its mouse orthologues, human Mx1 locates in the cytoplasm and is effective against a broad range of viruses, regardless their replication site $(43,44)$. To the current date, there is no reported inhibition of mammarenaviruses by $\mathrm{Mx} 1$. Nevertheless, in light of our results, further investigation is required to elucidate the mechanism of action of ISG15 and Mx1 against Mammarenavirus infection, and their biological implications. The different consequences of PKR on Mx1, ISG15 
and CCL5 expression kinetics suggests that, although all these genes are overall upregulated upon viral infections, they are also differently tuned by additional host factors such as PKR.

A complete vision of the innate immune response and its modulation is crucial to understand the mechanisms underlying the pathogenicity of mammarenaviruses, and for the development of new antiviral strategies. The results presented here contribute to a better understanding of the dynamics in the onset of the innate immune response triggered by NW mammarenavirus infections and, in particular, of the key role of PKR on it. The change in the kinetic and temporal expression patterns of Mx1 and ISG15, but not of CCL5, demonstrates that PKR does not only modulate the amplitude of the IFN-I response but also tunes the expression of selected ISGs over the time throughout mammarenavirus infection.

Author Contributions: The following statements should be used "Conceptualization, S.K. and H.M.; methodology, H.M..; validation, H.M..; formal analysis, H.M.; investigation, H.M.; resources, S.K. and H.M.; data curation, H.M..; writing - original draft preparation, H.M.; writing - review and editing, H.M.; visualization, H.M.; supervision, H.M.; project administration, H.M.; funding acquisition, S.K. Author Prof. Stefan Kunz was unable to confirm their authorship contributions. On their behalf, the corresponding author has reported their contributions to the best of their knowledge.

Funding: This research was supported by Swiss National Science Foundation grant SINERGIA Nr. CRSII3_160780/1 to S.K. and funds to S.K. from the University of Lausanne.

Data Availability Statement: All relevant data are within the manuscript.

Acknowledgments: Tragically, Stefan Kunz passed away on 10 of January of 2020. His memory will remain in among all of us who shared part of our lives and enjoyed his company. Because his enthusiasm, dedication, and motivation, he will be remembered as a wonderful person and scientist. We would like to thank Roberto Balbontín for his valuable and critical scientific feedback. We also would like to thank Gisa Gerold and Rebecca Møller for the A549/Scr and A549/PKR cells. The following reagent was obtained through BEI Resources, NIAID, NIH: monoclonal anti-Junin virus, clone MA03-BE06, NR-41860. This research was supported by Swiss National Science Foundation grant SINERGIA Nr. CRSII3_160780/1 to S.K. and funds to S.K. from the University of Lausanne.

Conflicts of Interest: The authors declare no conflict of interest.

\section{References}

1. Radoshitzky SR, Bao Y, Buchmeier MJ, Charrel RN, Clawson AN, Clegg CS, et al. Past, present, and future of arenavirus taxonomy. Arch Virol. 2015;160(7):1851-74.

2. Geisbert TW, Jahrling PB. Exotic emerging viral diseases: progress and challenges. Nat Med. 2004;10(12 Suppl):S110-21.

3. Buchmeier MJ, de la Torre JC, Peters C. Arenaviridae: the viruses and their replication. Fields Virology. 4 ed.: Philadelphia: Lippincott-Raven; 2007. p. 1791-828.

4. Centers for Disease C, Prevention. Fatal illnesses associated with a new world arenavirus--California, 1999-2000. MMWR Morb Mortal Wkly Rep. 2000;49(31):709-11.

5. Moreno H, Rastrojo A, Pryce R, Fedeli C, Zimmer G, Bowden TA, et al. A novel circulating tamiami mammarenavirus shows potential for zoonotic spillover. PLoS Negl Trop Dis. 2020;14(12):e0009004.

6. de la Torre JC. Molecular and cell biology of the prototypic arenavirus LCMV: implications for understanding and combating hemorrhagic fever arenaviruses. Ann N Y Acad Sci. 2009;1171 Suppl 1:E57-64.

7. Iwasaki M, Ngo N, Cubitt B, de la Torre JC. Efficient Interaction between Arenavirus Nucleoprotein (NP) and RNA-Dependent RNA Polymerase (L) Is Mediated by the Virus Nucleocapsid (NP-RNA) Template. J Virol. 2015;89(10):5734-8.

8. Knopp KA, Ngo T, Gershon PD, Buchmeier MJ. Single nucleoprotein residue modulates arenavirus replication complex formation. mBio. 2015;6(3):e00524-15. 
9. Hastie KM, Kimberlin CR, Zandonatti MA, MacRae IJ, Saphire EO. Structure of the Lassa virus nucleoprotein reveals a dsRNAspecific 3' to 5' exonuclease activity essential for immune suppression. Proc Natl Acad Sci U S A. 2011;108(6):2396-401.

10. Huang C, Kolokoltsova OA, Yun NE, Seregin AV, Ronca S, Koma T, et al. Highly Pathogenic New World and Old World Human Arenaviruses Induce Distinct Interferon Responses in Human Cells. J Virol. 2015;89(14):7079-88.

11. Jiang X, Huang Q, Wang W, Dong H, Ly H, Liang Y, et al. Structures of arenaviral nucleoproteins with triphosphate dsRNA reveal a unique mechanism of immune suppression. J Biol Chem. 2013;288(23):16949-59.

12. Martinez-Sobrido L, Giannakas P, Cubitt B, Garcia-Sastre A, de la Torre JC. Differential inhibition of type I interferon induction by arenavirus nucleoproteins. J Virol. 2007;81(22):12696-703.

13. Huang Q, Shao J, Lan S, Zhou Y, Xing J, Dong C, et al. In vitro and in vivo characterizations of pichinde viral nucleoprotein exoribonuclease functions. J Virol. 2015;89(13):6595-607.

14. Moreno H, Moller R, Fedeli C, Gerold G, Kunz S. Comparison of the Innate Immune Responses to Pathogenic and Nonpathogenic Clade B New World Arenaviruses. J Virol. 2019;93(19).

15. Rodrigo WW, Ortiz-Riano E, Pythoud C, Kunz S, de la Torre JC, Martinez-Sobrido L. Arenavirus nucleoproteins prevent activation of nuclear factor kappa B. J Virol. 2012;86(15):8185-97.

16. Xing J, Ly H, Liang Y. The Z proteins of pathogenic but not nonpathogenic arenaviruses inhibit RIG-I-like receptor-dependent interferon production. J Virol. 2015;89(5):2944-55.

17. Huang C, Kolokoltsova OA, Yun NE, Seregin AV, Poussard AL, Walker AG, et al. Junin virus infection activates the type I interferon pathway in a RIG-I-dependent manner. PLoS Negl Trop Dis. 2012;6(5):e1659.

18. Huang C, Kolokoltsova OA, Mateer EJ, Koma T, Paessler S. Highly Pathogenic New World Arenavirus Infection Activates the Pattern Recognition Receptor Protein Kinase R without Attenuating Virus Replication in Human Cells. J Virol. 2017;91(20).

19. King BR, Hershkowitz D, Eisenhauer PL, Weir ME, Ziegler CM, Russo J, et al. A Map of the Arenavirus Nucleoprotein-Host Protein Interactome Reveals that Junin Virus Selectively Impairs the Antiviral Activity of Double-Stranded RNA-Activated Protein Kinase (PKR). J Virol. 2017;91(15).

20. McAllister CS, Toth AM, Zhang P, Devaux P, Cattaneo R, Samuel CE. Mechanisms of protein kinase PKR-mediated amplification of beta interferon induction by C protein-deficient measles virus. J Virol. 2010;84(1):380-6.

21. McAllister CS, Taghavi N, Samuel CE. Protein Kinase PKR Amplification of Interferon beta Induction Occurs through Initiation Factor eIF-2 alpha-mediated Translational Control. Journal of Biological Chemistry. 2012;287(43):36384-92.

22. Gilfoy FD, Mason PW. West nile virus-induced interferon production is mediated by the double-stranded RNA-Dependent protein kinase PKR. Journal of Virology. 2007;81(20):11148-58.

23. Yang YL, Reis LF, Pavlovic J, Aguzzi A, Schafer R, Kumar A, et al. Deficient signaling in mice devoid of double-stranded RNAdependent protein kinase. EMBO J. 1995;14(24):6095-106.

24. Dey M, Cao C, Dar AC, Tamura T, Ozato K, Sicheri F, et al. Mechanistic link between PKR dimerization, autophosphorylation, and elF2 alpha substrate recognition. Cell. 2005;122(6):901-13.

25. Mateer EJ, Maruyama J, Card GE, Paessler S, Huang C. Lassa Virus, but Not Highly Pathogenic New World Arenaviruses, Restricts Immunostimulatory Double-Stranded RNA Accumulation during Infection. J Virol. 2020;94(9).

26. Mateer EJ, Paessler S, Huang C. Visualization of Double-Stranded RNA Colocalizing With Pattern Recognition Receptors in Arenavirus Infected Cells. Front Cell Infect Microbiol. 2018;8:251.

27. Sanchez A, Pifat DY, Kenyon RH, Peters CJ, McCormick JB, Kiley MP. Junin virus monoclonal antibodies: characterization and cross-reactivity with other arenaviruses. J Gen Virol. 1989;70 ( Pt 5):1125-32.

28. Kim S, Koo T, Jee HG, Cho HY, Lee G, Lim DG, et al. CRISPR RNAs trigger innate immune responses in human cells. Genome Research. 2018;28(3):367-73. 
29. Wienert B, Shin J, Zelin E, Pestal K, Corn JE. In vitro-transcribed guide RNAs trigger an innate immune response via the RIGI pathway. PLoS Biol. 2018;16(7):e2005840.

30. Schulz O, Pichlmair A, Rehwinkel J, Rogers NC, Scheuner D, Kato H, et al. Protein kinase R contributes to immunity against specific viruses by regulating interferon mRNA integrity. Cell Host Microbe. 2010;7(5):354-61.

31. Schall TJ, Bacon K, Toy KJ, Goeddel DV. Selective attraction of monocytes and T lymphocytes of the memory phenotype by cytokine RANTES. Nature. 1990;347(6294):669-71.

32. Kim MO, Suh HS, Brosnan CF, Lee SC. Regulation of RANTES/CCL5 expression in human astrocytes by interleukin-1 and interferon-beta. J Neurochem. 2004;90(2):297-308.

33. Matloubian M, Concepcion RJ, Ahmed R. CD4+ T cells are required to sustain CD8+ cytotoxic T-cell responses during chronic viral infection. J Virol. 1994;68(12):8056-63.

34. Scott EP, Aronson JF. Cytokine patterns in a comparative model of arenavirus haemorrhagic fever in guinea pigs. Journal of General Virology. 2008;89:2569-79.

35. Zhang D, Zhang DE. Interferon-stimulated gene 15 and the protein ISGylation system. J Interferon Cytokine Res. 2011;31(1):119-30.

36. Morales DJ, Lenschow DJ. The antiviral activities of ISG15. J Mol Biol. 2013;425(24):4995-5008.

37. Lindner HA, Lytvyn V, Qi H, Lachance P, Ziomek E, Menard R. Selectivity in ISG15 and ubiquitin recognition by the SARS coronavirus papain-like protease. Arch Biochem Biophys. 2007;466(1):8-14.

38. Mielech AM, Kilianski A, Baez-Santos YM, Mesecar AD, Baker SC. MERS-CoV papain-like protease has deISGylating and deubiquitinating activities. Virology. 2014;450:64-70.

39. Swatek KN, Aumayr M, Pruneda JN, Visser LJ, Berryman S, Kueck AF, et al. Irreversible inactivation of ISG15 by a viral leader protease enables alternative infection detection strategies. Proc Natl Acad Sci U S A. 2018;115(10):2371-6.

40. Zhang X, Bogunovic D, Payelle-Brogard B, Francois-Newton V, Speer SD, Yuan C, et al. Human intracellular ISG15 prevents interferon-alpha/beta over-amplification and auto-inflammation. Nature. 2015;517(7532):89-93.

41. Zhao C, Sridharan H, Chen R, Baker DP, Wang S, Krug RM. Influenza B virus non-structural protein 1 counteracts ISG15 antiviral activity by sequestering ISGylated viral proteins. Nat Commun. 2016;7:12754.

42. Dittmann J, Stertz S, Grimm D, Steel J, Garcia-Sastre A, Haller O, et al. Influenza A virus strains differ in sensitivity to the antiviral action of Mx-GTPase. J Virol. 2008;82(7):3624-31.

43. Frese M, Kochs G, Feldmann H, Hertkorn C, Haller O. Inhibition of bunyaviruses, phleboviruses, and hantaviruses by human MxA protein. Journal of Virology. 1996;70(2):915-23.

44. Gordien E, Rosmorduc O, Peltekian C, Garreau F, Brechot C, Kremsdorf D. Inhibition of hepatitis B virus replication by the interferon-inducible MxA protein. Journal of Virology. 2001;75(6):2684-91.

45. Kochs G, Haller O. Interferon-induced human MxA GTPase blocks nuclear import of Thogoto virus nucleocapsids. Proc Natl Acad Sci U S A. 1999;96(5):2082-6.

46. Reichelt M, Stertz S, Krijnse-Locker J, Haller O, Kochs G. Missorting of LaCrosse virus nucleocapsid protein by the interferoninduced MxA GTPase involves smooth ER membranes. Traffic. 2004;5(10):772-84. 